\title{
Desarrollo competencial del alumno: el espíritu emprendedor y la responsabilidad social
}

\author{
Tiziana Priede Bergamini ${ }^{a}$, Cristina López-Cózar Navarro ${ }^{b}$ y Sonia Benito \\ Hernández ${ }^{c}$
}

${ }^{a}$ Profesora de la Universidad Europea de Madrid tiziana.priede@universidadeuropea.es, ${ }^{b}$ Profesora de la Universidad Politécnica de Madrid cristina.lopezcozar@upm.es y ${ }^{c}$ Profesora de la Universidad Politécnica de Madrid sonia.benito@upm.es.

\begin{abstract}
In order to achieve a business framework in which companies are able to respond adequately to the economic, social and environmental challenges; it is necessary to take a step further in higher education. Accordingly, the university teaching staff must assume the commitment to contribute to the development of competences, not only linked with entrepreneurial spirit, but also with social responsibility and ethical commitment at work. All those proposals that aim to bring students to responsible, sustainable and committed business initiatives with the community acquire a special relevance today. For this reason, this paper presents an interesting teaching experience for university students trying to stimulate their curiosity for social entrepreneurship, and at the same time, bringing them closer to the business reality.
\end{abstract}

Keywords: Entrepreneurial spirit, responsible behavior, social enterprise, sustainability, training, higher education, skills.

\begin{abstract}
Resumen
Con el objetivo de conseguir un tejido productivo en el que las empresas sean capaces de responder adecuadamente a los retos económicos, sociales y medioambientales, es preciso dar un paso más en la formación universitaria. En este sentido, el profesorado debe asumir el compromiso de contribuir al desarrollo de competencias relacionadas, no solo con el espíritu emprendedor, sino también con la responsabilidad social y el compromiso ético en el trabajo. Todas aquellas propuestas que pretendan acercar al alumnado a iniciativas empresariales responsables, sostenibles $y$ comprometidas con la comunidad adquieren una especial relevancia en el momento actual. Por ello, en el presente trabajo se expone una experiencia educativa interesante para el alumnado de educación superior, con la que se pretende estimular su curiosidad por el emprendimiento social, así como su acercamiento a la realidad empresarial.
\end{abstract}

Palabras clave: espíritu emprendedor, comportamiento responsable, empresa social, sostenibilidad, formación, educación superior, competencias. 


\section{Introducción}

La implantación del Espacio Europeo de Educación Superior (EEES) ha proporcionado el marco adecuado para lograr un desarrollo competencial integral del alumnado. Entre las competencias que se pueden encontrar en los planes de estudios de los grados universitarios suele hacerse alusión al fomento del emprendimiento. Se trata de una competencia muy amplia, que puede adoptar diferentes denominaciones tales como iniciativa, capacidad emprendedora o espíritu emprendedor. En el presente trabajo se ha optado por esta última denominación, coincidiendo tanto con el informe "Educación y Formación 2010" de la Comisión Europea, en el que se le otorga la importancia de competencia clave; con el informe elaborado por Accenture y Universia (2007), así como con en el Libro Blanco de Economía y Empresa (ANECA), el cual la incluye dentro de las competencias genéricas sistémicas.

Por otra parte, también es frecuente hallar referencias sobre la importancia de desarrollar en los estudiantes el compromiso ético y la responsabilidad social en el trabajo. Así por ejemplo, en la recomendación 2006/962/CE del Parlamento Europeo y del Consejo, de 18 de diciembre de 2006, sobre las competencias clave para el aprendizaje permanente, se señala que este espíritu empresarial debe implicar una concienciación sobre los valores éticos e impulsar la buena gobernanza. Asimismo, entre las competencias clave incluye la social y la cívica, y se señala que las personas deben interesarse por el desarrollo socioeconómico sostenible, la diversidad de valores, el apoyo a la cohesión social y estar dispuestas a comprometerse. Por su parte, en el citado Libro Blanco de la ANECA, entre las competencias genéricas personales se incluye el compromiso ético en el trabajo.

Actualmente en nuestro país, tras una larga situación de crisis económica y de valores, se debaten nuevos modelos de desarrollo, con el fin de lograr la recuperación y un futuro crecimiento sostenible. Ante este panorama, consideramos que es especialmente importante que los jóvenes universitarios adquieran estas dos competencias de manera conjunta: el espíritu emprendedor y la responsabilidad social unida al comportamiento ético en el trabajo. Hoy más que nunca, es preciso transmitir a nuestros estudiantes que las empresas deben modificar sus actitudes y comportamientos. Por tanto, es fundamental fomentar en las aulas el espíritu emprendedor responsable, con el propósito de conseguir un tejido empresarial en el que los nuevos proyectos empresariales sean capaces de asegurar su viabilidad económica y, al mismo tiempo, avanzar hacia una mayor integración social, mayor respeto medioambiental y un modelo de crecimiento más coherente y equilibrado.

\section{Objetivos}

En los últimos años es notable el interés que el emprendimiento social ha despertado como reflejo del importante desarrollo que estas iniciativas están experimentando (Santos, 2012), por lo que la comunidad universitaria no debería permanecer ajena a esta realidad. Consideramos, pues, que resulta muy oportuno ampliar el conocimiento existente para contribuir a comprender mejor el concepto de emprendedor social, sus peculiaridades y sus aspectos diferenciadores. Se trata, en general, de ofrecer a los estudiantes una visión global

(c)) BY-NC-ND 2017, Universitat Politècnica de València 
de manera que contemplen este tipo de emprendimiento como una alternativa económica, social y laboral interesante para su futuro profesional. Por ello, el presente trabajo plantea una propuesta educativa interesante para los universitarios intentando estimular su curiosidad por el emprendimiento social, así como su acercamiento a la realidad empresarial, considerando que el deporte ofrece una excelente oportunidad para el desarrollo de estas iniciativas innovadoras.

Se presenta, por tanto, una experiencia de innovación docente claramente orientada a la formación en competencias del estudiante, cuyos principales objetivos son: promover el compromiso social mediante el aprendizaje activo; fomentar el emprendimiento ligado a un proyecto sostenible y responsable en el ámbito deportivo; acercar al alumnado a la realidad empresarial y a las prácticas empresariales responsables; y ofrecer nuevas alternativas profesionales. Dicha experiencia ha sido realizada con estudiantes del Grado en Gestión Deportiva -a través de la asignatura de Economía de la Empresa- durante el curso 20152016.

Con el fin de describir el desarrollo de la actividad propuesta para alcanzar estos objetivos, el presente trabajo se estructura de la siguiente manera: en primer lugar, en cuanto al desarrollo de la innovación, se presenta el contexto en el que se lleva a cabo la actividad; se realiza una aproximación al concepto de emprendedor social y su aplicación en el entorno deportivo a través del cual es posible la consecución de objetivos sociales en diferentes ámbitos, tales como la salud, la integración o la educación, entre otros. Así mismo, se plantean las competencias que se pretenden desarrollar y se presenta la descripción de la propuesta. En el siguiente epígrafe se muestran los resultados obtenidos de la encuesta de valoración realizada al grupo de estudiantes que ha participado en la experiencia. El análisis de estos datos permitirá verificar si se han alcanzado los objetivos iniciales propuestos. Finalmente, se procede a exponer las principales conclusiones extraídas del trabajo.

\section{Desarrollo de la innovación}

\subsection{Contextualización de la actividad}

En la actualidad, una de las competencias más demandadas por la sociedad a sus jóvenes graduados es el espíritu emprendedor (Toledano, 2006; López-Cózar y Priede, 2009; Benavides et al., 2010; Priede et al, 2013; Juliá, 2013). No obstante, como ya se ha dicho anteriormente, esta competencia debería estar sustentada sobre una base de valores compartidos. En efecto, lamentablemente hoy en día es frecuente encontrar empresarios que presentan importantes carencias de ética y principios a la hora de tomar decisiones llevar a cabo sus negocios. Es recomendable, por lo tanto, fomentar en los jóvenes el emprendimiento ligado a un proyecto responsable y sostenible, que aporte valor global. El profesorado debe, no sólo desarrollar competencias sino también contribuir a la formación de buenos ciudadanos (Marina, 2009), por lo que resulta interesante impulsar esta actitud emprendedora dentro del ámbito de la empresa social (Fisac et al., 2011; Lejarriaga, et al., 2013; Martín et al., 2013; López-Cózar y Priede, 2014). Como se señala en la citada 
recomendación del Parlamento Europeo (2006), "las personas deben ser conscientes también de la postura ética de las empresas y de cómo éstas pueden ser un impulso positivo, por ejemplo, mediante el comercio justo y las empresas sociales". Consideramos, pues, que el espíritu emprendedor debe desarrollarse desde el lado más humano, buscando el compromiso social para alcanzar un progreso continuo, justo y equitativo.

El emprendedor social es aquel que reconoce una oportunidad de negocio buscando la resolución creativa de un problema social o medioambiental. Se trata de una persona con un marcado carácter innovador, que posee un gran sentido de la responsabilidad y un claro compromiso con la comunidad. Este fuerte compromiso se materializa en el desarrollo de una iniciativa empresarial motivada por la voluntad de ayudar a los demás y de llevar a cabo un cambio en la sociedad. Como señala Bornstein (2005), el emprendedor social es aquel que es capaz de identificar recursos allí donde los demás sólo ven dificultades. No se trata de filantropía o caridad, al contrario, deben ser negocios rentables, si bien, la reinversión de los beneficios económicos en su propio objetivo favorece que estas organizaciones sean idóneas para impulsar a las comunidades a las que pertenecen. En definitiva, consiste en la creación de un modelo de negocio que permita descubrir, definir y aprovechar las oportunidades para incrementar la riqueza social (Mair y Martí, 2006; Zahra et al, 2009; Defourny y Nyssens, 2012; Defourny et al., 2014; Priede et al., 2014).

Por su parte, este tipo de emprendimiento encuentra en el ámbito deportivo diversas oportunidades ya que el deporte puede ser empleado como vía para fomentar soluciones a determinados problemas sociales. El término deporte es un concepto abierto que se refiere a una amplia variedad de actividades, que pueden ser practicadas con diferentes objetivos (competición, ocio, salud, etc.) y que abarca múltiples aspectos de diferentes ámbitos (educativo, social, económico, etc.). La práctica deportiva como aficionado es básica en la sociedad moderna, ya que puede desarrollar la salud, la integración social, la educación, etc. Diversas investigaciones realizadas sobre la relación entre el deporte y la salud, han demostrado los efectos beneficiosos de la práctica continuada de actividades deportivas (World Health Organization, 2003).

Al mismo tiempo, el deporte es un elemento de cohesión y relación social, que ayuda al desarrollo de la personalidad y al establecimiento de relaciones entre sus practicantes, con el resultado positivo que ello implica. Por tanto, además de los efectos sobre la salud, el deporte supone educación -el deporte inculca valores- e integración social (Carranza y Maza, 2011; del Arco y Rodríguez, 2013; López-Cózar et al., 2015). Así pues, a través del deporte se pueden plantean actividades para diversos colectivos con necesidades específicas como, por ejemplo, personas con discapacidad, personas en situación de maltrato, desempleados de larga duración, entre otros. Los emprendedores sociales pueden actuar en la comunidad deportiva de manera innovadora con el fin de crear un beneficio económico y social. De este modo, el deporte y el emprendimiento social persiguen objetivos semejantes como la cohesión y la integración, junto con el logro de una sociedad mejor para todos, dentro de un proyecto responsable en todas sus dimensiones, por lo que pueden considerarse buenos aliados. 


\subsection{Capacidades desarrolladas con la actividad}

La actividad planteada permite a los estudiantes desarrollar diversas competencias establecidas en el ámbito de educación superior:

- Espíritu emprendedor: observación de la realidad económica y social, detección de oportunidades para la puesta en marcha de un negocio vinculado a un problema social con el fin de promover, la integridad, la sostenibilidad, la inclusión, etc.

- Responsabilidad social: comportamiento responsable, ético y comprometido.

- Aprendizaje activo: el alumnado se sitúa en el centro de su propio aprendizaje, participando, reflexionando y desarrollando el pensamiento crítico. Conocer desde la experiencia.

- Innovación y creatividad: esfuerzo por presentar algo diferente, por buscar alternativas no exploradas. Presentación de ideas de forma atractiva e interesante.

- Trabajo en equipo y cooperación: se forman equipos de trabajo para realizar la actividad. El alumnado debe cooperar para aprender.

- Habilidades comunicativas: saber transmitir las principales ideas y explicar la esencia del trabajo realizado, hacerlo además de forma atractiva e interesante.

\subsection{Descripción de la actividad}

La actividad comienza con una sesión teórica en la que el docente sensibiliza al alumnado sobre el concepto de emprendimiento social y empresa social, sus principales características y sus posibilidades de aplicación en el ámbito deportivo. Esta primera toma de contacto con el concepto es necesaria para sentar las bases que permitan desarrollar posteriormente el trabajo práctico con éxito.

A continuación, se constituyen los equipos de trabajo supervisados por el profesor con el fin de formar grupos homogéneos. Es importante la elección de un líder que acepte la figura de coordinador y un secretario que apoye su tarea. Se deja libremente al grupo la asignación de estos roles.

Posteriormente, el profesor proporciona a los equipos las pautas concretas de la actividad a desarrollar, la cual consiste en hacer un seguimiento de una empresa social del ámbito deportivo; dicho seguimiento puede hacerse sobre una empresa real ya existente en el mercado, o bien sobre una empresa ideada por los propios estudiantes. En cualquiera de las dos opciones, se proporciona un documento en el que se recogen las cuestiones que obligatoriamente deben ser tratadas. Igualmente, se indica a los estudiantes que deberán entregar un documento escrito donde se recoja el estudio realizado y se dé respuesta a las preguntas previamente planteadas, así como un breve video, lo más creativo posible, que recoja las principales conclusiones extraídas de su análisis. Tanto las pautas facilitadas por el profesor, como las entregas de los estudiantes estarán centralizadas en la plataforma virtual blackboard.

La parte final de la actividad consiste en la presentación de los resultados. Cada grupo deberá realizar una presentación oral con el fin de compartir la experiencia y el 
conocimiento con el resto del alumnado. En dicha presentación deben participar todos los miembros del equipo, que tienen que presentar un breve informe sobre la investigación realizada, además de proyectar el video elaborado. Tras la exposición, el docente dirige preguntas concretas a los diferentes componentes del grupo que le permitan comprobar el nivel de conocimiento global e individual, así como con el propósito de evitar que sólo aprendan la parte del trabajo que han realizado. Así mismo, el resto de los estudiantes debe plantear cuestiones al equipo que presenta el video con el fin de promover la generación de conocimiento conjunto, la cooperación y la participación de todos.

Respecto a la evaluación, el peso de esta actividad sobre la calificación global de la asignatura es del $20 \%$. Un $15 \%$ representa la nota del informe escrito y la elaboración del video, y es común a todos los integrantes del grupo, y un 5\% corresponde a la presentación oral que es una nota individual para cada estudiante.

\section{Resultados}

Una vez finalizada la actividad, se realizó una encuesta a los estudiantes para conocer su opinión sobre el grado de desarrollo de las competencias que se estaban trabajando, así como su nivel de satisfacción con la misma. El grupo estaba compuesto por 18 estudiantes, de los que 13 contestaron a la encuesta ( $72 \%$ de respuesta), lo cual se puede considerar una alta participación. En la tabla 1 se presentan las cuestiones planteadas y su relación con los objetivos y competencias objeto del estudio.

Tabla 1. Preguntas de la encuesta

\begin{tabular}{|c|c|c|}
\hline Pregunta encuesta & Objetivos & Competencias \\
\hline $\begin{array}{l}\text { ¿Considera que esta actividad ha } \\
\text { despertado su interés por el } \\
\text { emprendimiento social y las } \\
\text { prácticas empresariales responsables? }\end{array}$ & $\begin{array}{l}\text { Acercar al alumnado a la } \\
\text { realidad empresarial y a las } \\
\text { prácticas empresariales } \\
\text { responsables. }\end{array}$ & Espíritu emprendedor \\
\hline $\begin{array}{l}\text { ¿Considera que esta actividad le ha } \\
\text { enseñado nuevas alternativas para } \\
\text { su futuro profesional? }\end{array}$ & $\begin{array}{l}\text { Ofrecer nuevas alternativas } \\
\text { profesionales. }\end{array}$ & Espíritu emprendedor \\
\hline $\begin{array}{l}\text { ¿Considera que con esta actividad se } \\
\text { ha incrementado su propio } \\
\text { compromiso social? }\end{array}$ & $\begin{array}{l}\text { Promover el compromiso } \\
\text { social mediante el } \\
\text { aprendizaje activo. }\end{array}$ & Responsabilidad social \\
\hline $\begin{array}{l}\text { ¿Considera que con esta actividad } \\
\text { ha despertado su espíritu } \\
\text { emprendedor? }\end{array}$ & $\begin{array}{ll}\text { Fomentar } & \mathrm{el} \\
\text { emprendimiento ligado } & \mathrm{a} \\
\text { un proyecto sostenible } & \mathrm{y} \\
\text { responsable. } & \end{array}$ & Espíritu emprendedor \\
\hline $\begin{array}{l}\text { ¿Considera que con esta actividad } \\
\text { ha desarrollado la competencia de } \\
\text { trabajo en equipo? }\end{array}$ & $\begin{array}{l}\text { Promover el compromiso } \\
\text { social mediante el } \\
\text { aprendizaje activo. }\end{array}$ & $\begin{array}{l}\text { Trabajo en equipo y } \\
\text { cooperación }\end{array}$ \\
\hline
\end{tabular}

(cc) EY-NC-ND 2017, Universitat Politècnica de València 


\begin{tabular}{|c|c|c|}
\hline $\begin{array}{l}\text { ¿Considera que con esta actividad } \\
\text { promueve el aprendizaje activo } \\
\text { (aprender con las experiencias)? }\end{array}$ & $\begin{array}{l}\text { Promover el compromiso } \\
\text { social mediante el } \\
\text { aprendizaje activo. }\end{array}$ & Aprendizaje activo \\
\hline $\begin{array}{l}\text { ¿Considera que con esta actividad se } \\
\text { han aplicado los } \\
\text { adquiridos a conocimientos } \\
\text { asignatura? }\end{array}$ & $\begin{array}{l}\text { Promover el compromiso } \\
\text { social mediante el } \\
\text { aprendzaje activo. }\end{array}$ & \\
\hline $\begin{array}{l}\text { ¿Considera que con esta actividad } \\
\text { ha desarrollado las competencias de } \\
\text { creatividad e innovación? }\end{array}$ & & Innovación y creatividad \\
\hline $\begin{array}{l}\text { ¿Considera que con esta actividad } \\
\text { ha desarrollado la competencia de } \\
\text { comunicación oral? }\end{array}$ & & Habilidades comunicativas \\
\hline $\begin{array}{l}\text { Nivel de satisfacción global con la } \\
\text { actividad desarrollada }\end{array}$ & & \\
\hline
\end{tabular}

Fuente: Elaboración propia

Por su parte, en la figura 1 se muestran las frecuencias de los resultados obtenidos empleando una escala Likert 1 (muy bajo) a 5 (muy alto) para cada una de las cuestiones que se plantearon.

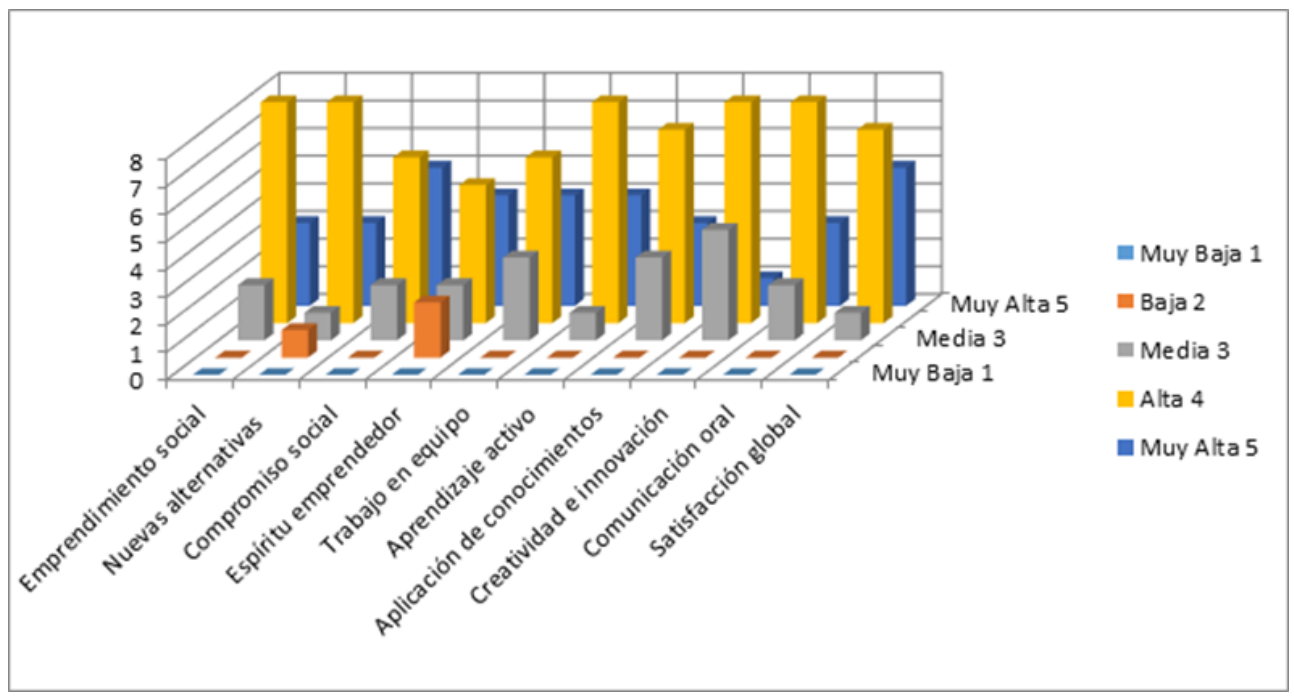

Fig. 1 Frecuencias de los resultados obtenidos

Como puede observarse en la figura 1, la mayoría de los estudiantes valoró la actividad muy positivamente, mostrando alta satisfacción con el aprendizaje obtenido, el desarrollo de compencias y, en general, con la utilidad de la actividad para su formación. 


\section{Conclusiones}

En el presente trabajo se ha expuesto una actividad formativa llevada a cabo con los alumnos de la carrera de gestión deportiva durante el curso 2015-2016, con la que se ha pretendido desarrollar conjuntamente el espíritu emprendedor y la responsabilidad social unida al comportamiento ético en el trabajo, dos competencias que presentan especial relevancia en un contexto económico y social como el actual, en el que es preciso la búsqueda de un modelo de crecimiento sostenible, más justo y equitativo. Es precisamente en las aulas universitarias donde los docentes tenemos la oportunidad de poder alcanzar a nuestros jóvenes y orientarlos en esta dirección, lo que ha inspirado sin duda la realización de esta experiencia.

Las propuestas presentadas por los estudiantes fueron tres: la creación de una empresa social denominada Bizitza (vida), cuya actividad es una academia de deportes para promover la igualdad de los niños con discapacidad; el seguimiento de la empresa de orientación social Vaude de ropa y equipamiento para deportes de montaña en la que se combina la sostenibilidad e innovación; y el seguimiento de la empresa social Senda, la cual se dedica a la fabricación y comercialización -a través del comercio justo- de balones y equipamiento deportivo. Los tres ejemplos presentados fueron de gran interés $y$, cada uno en su estilo, resultaron generadores de debate y opinión, además de servir de ejemplos para la posible orientación del futuro profesional de estos estudiantes.

Tras el análisis de los resultados de la encuesta de satisfacción realizada a los propios implicados, se desprende que efectivamente consideran que se trata de una actividad formativa que promueve el aprendizaje activo y con la que se pueden desarrollar las competencias que les permitan la consecución de un mundo más equilibrado y responsable, en el ejercicio de su futuro profesional. Concretamente podermos afirmar que se ha conseguido: aplicar el aprendizaje activo; fomentar el emprendimiento social y responsable en el ámbito deportivo; fomentar el compromiso social; desarrollar diversas competencias; aplicar conocimientos y ofrecer alternativas profesionales.

La tendencia en todos los sistemas educativos europeos, incluyendo los distintos niveles, debe encamimarse hacia el desarrollo de actitudes y comportamientos de estas características con el fin de formar ciudadanos con empuje, capaces de generar empleo y riqueza, y a la vez también capaces de llevar a cabo sus actividades de manera ética, responsable y sostenible. En este sentido, el emprendimiento social se configura como una alternativa factible y adaptable al orden económico actual, capaz de combinar la consecución de objetivos económicos, medioambientales y sociales, tres elementos clave de la sostenibilidad empresarial citados por las Naciones Unidas el Global Reporting Initiative (Izquierdo y Grañana, 2005).

Si bien actualmente es habitual encontrar en las universidades diversas propuestas que fomentan el espíritu emprendedor entre el alumnado, son todavía escasas las iniciativas que centran de manera concreta en el emprendimiento social. Consideramos que el desarrollo de iniciativas como la planteada a lo largo de este trabajo, consiguen estimular el espíritu

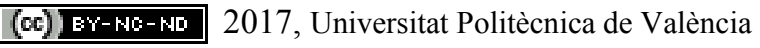


emprendedor y fomentar al mismo tiempo una actitud responsable hacia la sociedad en general.

\section{Referencias}

ACCENTURE Y UNIVERSIA (2007). Las competencias profesionales en los titulados. Contraste y diálogo Universidad-Empresa. Presentación final de resultados. Disponible: http://www.unizar.es/ice/images/stories/calidad/EstudioCompleto.pdf [Consulta: 22 de febrero de 2017].

Alemany, L., ÁlVAREZ, C., PlANEllas, M. y URBANO, D. (2011). Libro Blanco de la iniciativa emprendedora en España. Barcelona: Fundación Príncipe de Gerona ESADE.

BENAVIDES, M., SÁNCHEZ, I. y LUNA-AROCAS, R. (2010). "El proceso de aprendizaje para los emprendedores en la situación actual; un análisis cualitativo en el ámbito universitario". Revista de Dirección, Organización y Administración de Empresas, vol. 30 , p. 34-48.

BORNSTEIN, D. (2005). Cómo cambiar el mundo: los emprendedores sociales y el poder de las nuevas ideas. Barcelona: Editorial Debate.

CARRANZA, M. y MAZA, G. (Coords.) (2011). Deporte, actividad fisica e inclusión social. Una guía para la intervención social a través de las actividades deportivas. Madrid: Consejo Superior de Deportes.

CONSEJO DE LA UNIÓN EUROPEA (2004). "Educación y formación 2010" - Urgen las reformas para coronar con éxito la estrategia de Lisboa. Bruselas. Disponible: http://register.consilium.europa.eu/doc/srv?l=ES\&f=ST\%206905\%202004\%20INIT.

[Consulta: 10 de febrero de 2017].

DEFOURNY, J. y NYSSENS, M. (2012). "El enfoque EMES de la empresa social desde una perspectiva comparada". CIRIEC-España, Revista de Economía Pública, Social y Cooperativa, vol. 75, p. 7-34.

DEFOURNY, J., HULGÅRD, L. y PESTOFF, V. (2014). Social enterprise and the third sector: Changing European landscapes in a comparative perspective. Oxon: Routledge.

DEL ARCO, J. y RODRÍGUEZ, A. (2013). "Interrelación entre Actividad Deportiva y Desarrollo Socio-económico" en Revista Internacional de Deportes Colectivos, vol. 16, p. $12-21$.

FISAC, R., MORENO, A., PALACIOS, M., PEREZ, D. y URIBE, D. (2011). "La empresa social como modelo organizativo para la implicación de la universidad en iniciativas de cooperación al desarrollo" en V Congreso Universidad y Cooperación al Desarrollo. Cádiz 6/8 abril 2011. Disponible en linea (consulta 18 de enero de 2017). 
IZQUIERDO, R. S. y GRAÑANA, I. V. (2005). "La Responsabilidad Social Corporativa (RSC) y su gestión integrada”. CIRIEC-España, Revista de Economía Pública, Social y Cooperativa, vol. 53, p. 137-161.

JULIÁ, J. F. (2013). "Emprendimiento y universidad. Una referencia al caso de España ya la UPV”. REVESCO, Revista de Estudios Cooperativos, vol. 113, p. 7-27.

LEJARRIAGA, G., BEL, P. y MARTÍN, S. (2013). "El emprendimiento colectivo como salida laboral de los jóvenes: análisis del caso de las empresas de trabajo asociado" en REVESCO: Revista de Estudios Cooperativos, vol. 112, p. 36-65

LÓPEZ-CÓZAR, C. y PRIEDE, T. (2009). "La necesidad de un enfoque estratégico ante el planteamiento de los nuevos grados en el área de administración y dirección de empresas" en Revista de Docencia Universitaria, vol. 3, p. 1-14.

LÓPEZ-CÓZAR, C. y PRIEDE, T. (2014). "Una propuesta para acercar al alumnado al emprendimiento responsable y sostenible" en XI Jornadas Internacionales de Innovación Universitaria. Educar para transformar, Madrid: Universidad Europea de Madrid. 874881.

LÓPEZ-CÓZAR, C., PRIEDE, T. y DEL ARCO, J. (2015). "La empresa social. Experiencias innovadoras a través del deporte" en REVESCO: Revista de Estudios Cooperativos, vol. 119, p. 107-131.

MAIR, J. y MARTI, I. (2006). « Social entrepreneurship research: A source of explanation, prediction, and delight” en Journal of World Business, vol. 41, issue 1, p. 36-44.

MARINA, J. A. (2010). “La competencia de emprender”. Revista de Educación, 351, 4971.

MARTÍN, S., FERNÁNDEZ, J., BEL, P. y LEJARRIAGA, G. (2013). "Necesidad de medidas para impulsar la creación de las empresas de participación desde los diferentes niveles de enseñanza". CIRIEC-España, Revista de Economía Pública, Social y Cooperativa, vol. 78, p. 71-99.

PRIEDE, T., BENITO, S. y LÓPEZ-CÓZAR, C. (2013). “Cómo fomentar el espíritu emprendedor en las aulas universitarias. Descripción de una experiencia docente". Revista de Enseñanza Universitaria, vol. 39, p. 1-13.

PRIEDE, T., LÓPEZ-CÓZAR, C. y RODRÍGUEZ, A. (2014). “Análisis del marco económico-jurídico específico para los emprendedores sociales. Un estudio comparado entre diversos países". CIRIEC-España Revista de Economía Pública, Social y Cooperativa, vol. 80, p. 115-138.

SALINAS F. y OSORIO, L. (2012). "Emprendimiento y Economía Social, oportunidades y efectos en una sociedad en transformación". CIRIEC-España, Revista de Economía Pública, Social y Cooperativa, vol. 75, p. 128-151.

SANTOS, N.J. (2012). "A positive theory of social entrepreneurship". Journal of Business Ethics, Vol. 111, issue 3, p. 335-351.

(cc) EY-NC-ND 2017, Universitat Politècnica de València 
TOLEDANO, N. (2006). "Las perspectivas empresariales de los estudiantes universitarios" en Revista de Educación, vol. 341, p. 803-828.

WORLD HEALTH ORGANIZATION (2003). Health and development through physical activity and sport. Ginebra: World Health Organization Health Education and Health Promotion Unit.

ZAHRA, S.A., GEDAJlOVIC, E., NEUBAUM, D.O. y SHULMAN, J.M. (2009). "A typology of social entrepreneurs: Motives, search processes and ethical challenges" en Journal of Business Venturing, vol. 24, issue 5, p. 519-532. 